\title{
Molecular Model for Wall Slip: Role of Convective Constraint Release
}

\author{
Yogesh M. J oshi, ${ }^{\dagger}$ Ashish K. Lele, ${ }^{\dagger, \S}$ and R. A. Mashelkar*,‡ \\ Chemical Engineering Division, National Chemical Laboratory, Pune, 411 008, India; and Council of \\ Scientific and Industrial Research, Anusandhan Bhavan, 2 Rafi Marg, New Delhi, 110 001, India
}

Received J une 12, 2000; Revised Manuscript Received February 9, 2001

\begin{abstract}
A tube model is proposed for predicting wall slip phenomenon driven by the interfacial "disentanglement" mechanism. We model the dynamics of tethered chains, which are grafted on a highenergy wall and are entangled with the bulk chains flowing past them. The starting point of our model is the contour variable model (Mead; et al. Macromolecules 1998, 31, 7895), from which we develop a constitutive equation for tethered chains. We show that while the bulk chains are able to relax their orientation in the intermediate shear rate regi me by the convective constraint release (CCR) mechanism, the tethered chains experience a highly restricted CCR that is unable to randomize its flow-induced orientation above a critical shear rate or stress. This decreases the resistance to flow for the bulk chains, which suddenly slip past the oriented tether ed chains. The model correctly predicts the mol ecular weight dependence of the slip length, critical slip velocity and critical wall shear stress. It also quantitatively predicts the slip length and the critical slip velocity for a PDMS melt for which valuable molecular level experimental data are available in the literature.
\end{abstract}

\section{Introduction}

Slip instabilities observed during the shear flow of entangled polymeric liquids originate from the interfacial dynamics of tethered molecules at the wall. It is generally believed that chains, which are strongly tethered to a high-energy wall, disentangle from the bulk chains at a critical stress leading to discontinuous slip by a "disentanglement" mechanism. This picture was first conceptualized by Bergam. ${ }^{1}$ Since then several models, both phenomenological and molecular, have been proposed to quantify slip due to the disentanglement mechanism. ${ }^{2}$ In this work, we provide further molecular insights into the complex phenomenon of wall slip with an emphasis on modeling toward a quantitative prediction, something that has been lacking so far.

We summarize here the important mol ecular models for disentanglement-driven slip. In a seminal contribution, Brochard and de Gennes ${ }^{3}$ developed a scaling model for the flow of a polymer melt along a wall on which the chains of the same polymer are tethered and entangled with the bulk chains. The model was developed for the case of low grafting density of tethered chains in the so-called "mushroom" region, in which different tethered chains do not overlap. They showed that slip could occur discontinuously when the diameter of the deforming cylindrical coil of a tethered molecule decreases below the entanglement spacing so that the bulk chains suddenly disentangle from the tethered chains and slip. The Brochard-de Gennes model correlated the stick-slip transition to a "coil to stretch" transition of the tethered chain. Ajdari et al. ${ }^{4}$ estimated the friction encountered by a test chain when pulled by one end at a finite velocity through a stationary bulk. This picture is identical to the case of flow of an

\footnotetext{
† National Chemical Laboratory.

₹ Council of Scientific and Industrial Research.

$\S$ Current address: University of Cambridge, Chemical Engineering Department Pembroke Street, Cambridge CB2 3RA, U.K.

* Corresponding author. E-mail: dgcsir@csir.res.in.
}

entangled melt past a tethered chain when the frame of reference moves with the center of mass velocity of the bulk chains. They showed that, for a given molecular weight of the tethered chain, the magnitude of the jump in slip velocity increased on increasing the molecular weight of the bulk chains. Brochard et al. ${ }^{5}$ extended their earlier model to the case of higher grafting density of tethered chains. They showed that, above a threshold surface coverage, the velocity at which slip occurs increased linearly with the surface coverage. Recently Mhetar and $\operatorname{Archer}^{6}$ developed a scaling model for the case of the mushroom region. They considered the conformational changes of the tethered molecule under flow and predicted various friction laws in different slip velocity regions, which qualitatively agreed with their experimental data.

The molecular models for slip proposed so far are essentially based on scaling theories, which, while providing a molecular basis for wall slip, cannot quantitatively predict the slip data. In this paper, we propose a molecular constitutive equation based on the contour variable (CV) tube model ${ }^{7}$ for predicting slip on a highenergy wall. Our motivation is to develop an understanding of the molecular mechanisms that are responsible for slip due to disentanglement and to quantitatively predict various characteristic features of wall slip.

The CV model is a refined version of the original DoiEdwards tube model and takes into account most of the possible mechanisms of stress relaxation. The basic tube model considers a test chain trapped in a mesh (tube) of constraints, which restricts its lateral motion, while permitting it to move backward or forward only along its contour. Such snakelike motion along the contour is called reptation. If an instantaneous deformation is applied, it stretches the chain in the direction of the deformation. The stretched chain retracts back in a retraction time $\tau_{r}$ so as to restore the number of monomers per unit length of the original chain. Although the retraction process relaxes the stretch of the contour length, the chain still remains oriented in the 
direction of the deformation, which contributes to the stress. The chain relaxes (randomizes) its orientation by completely vacating the original tube principally by reptation. The time required for the chain to completely vacate its original tube by reptation is called the reptation time $\tau_{\mathrm{d}}$. In the original Doi-Edwards model, the retraction time was neglected and the molecule was assumed to have a constant contour length. Since the only mode of stress relaxation was reptation, the model predicted a nonmonotonic shear stress $\left(\sigma_{12}\right)$-shear rate $\left(\kappa_{12}\right)$ relationship for steady shearing flows; the maximum in stress occurs at a shear rate approximately equal to the reciprocal of reptation time. Marrucci and Grizzuti ${ }^{8}$ incorporated stretching of contour length in the original model but could not qualitatively improve the steady shear behavior of the model. The nonmonotonic flow curve predicted by the tube model indicates a constitutive instability of the bulk polymer, which was earlier considered to be responsible for spurt flow. ${ }^{9,10}$ However, it has now been proved beyond doubt that spurt flow is essentially an interfacial phenomenon, and that bulk instabilities (if any) in steady shear flows are not responsible for the stick-slip instability. ${ }^{11}$

The main drawback of the Doi-Edwards-MarrucciGrizzuti ${ }^{8}$ model (DEMG) was the absence of an effective relaxation mechanism in the shear rate region $\tau_{\mathrm{r}}{ }^{-1}>$ $\kappa_{12}>\tau_{\mathrm{d}}{ }^{-1}$. Marrucci ${ }^{12}$ made a significant conceptual advance by arguing that the missing mechanism was convective constraint release (CCR), which would occur due to the renewal of topological obstades through a relative motion between the tube and the chain due to flow. The CV model ${ }^{7}$ incorporates the mechanisms of CCR and contour length fluctuations in the DEMG model and successfully predicts steady shear experimental data over a large range of shear rates.

In this work, we use the CV model as a starting point and devel op a constitutive equation for the deformation of strongly adsorbed tethered chains that are entangled with flowing bulk chains. The model is described in section II of this paper, and it takes into account all of the essential molecular relaxation mechanisms for a tethered chain namely retraction, constraint release, convective constraint release and contour length fluctuations. Section III describes the salient predictions of the model and also demonstrates quantitative comparison with experimental data from theliterature. Section IV summarizes the conclusions of our work and discusses further scope for model development.

\section{Theoretical Section}

In a real extrusion system, polymer chains in the vicinity of a wall adsorb to form loops and tails, which are entangled with the free bulk chains. The bulk and the adsorbed chains have polydispersity in terms of molecular weights and loop sizes. In the present paper, we develop a tube model for the simplified case of monodi sperse end-tethered molecules of $\mathrm{N}$ segments per molecule, which are entangled with monodisperse bulk chains of $\mathrm{P}$ segments per molecule. We will call the grafted wall region as the "interfacial" region and the region away from the wall as the "bulk". We restrict our model devel opment to the mushroom regime at the wall in which the tethered chains do not overlap with each other. Thus, the tube constraining a tethered chain is formed only by the bulk chains. TheP chains of the bulk flow past the wall with a (unknown) slip velocity $V_{s}$ as shown in Figure la.
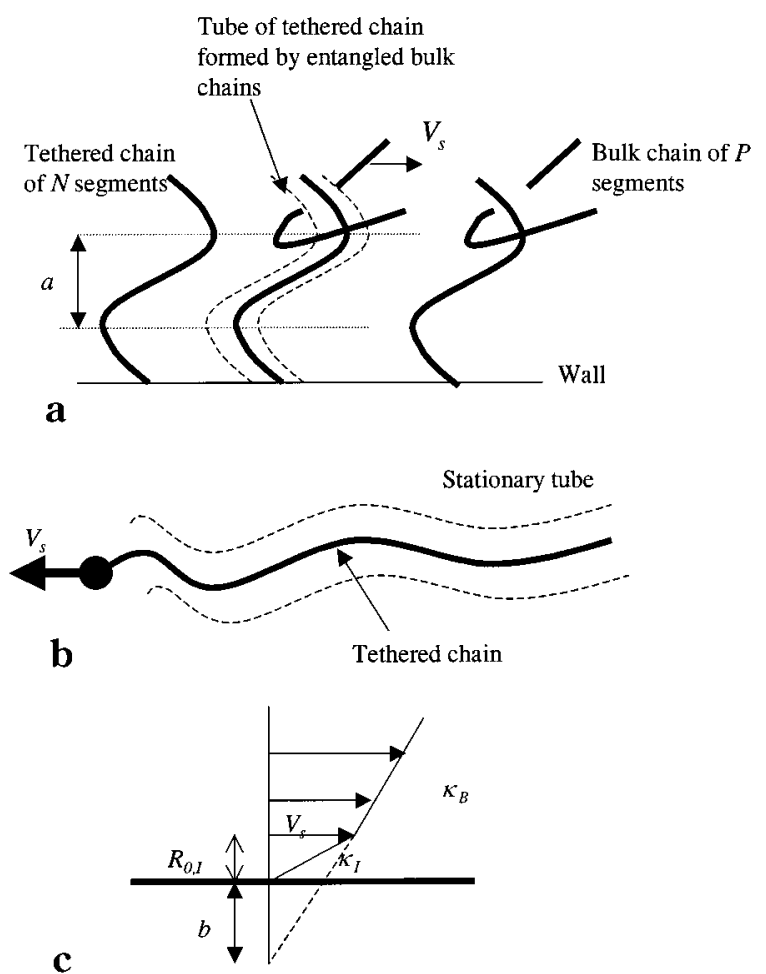

Figure 1. (a) Bulk chains flowing with macroscopic velocity $V_{s}$ entangled with the tethered chains. The tubes of the tethered chains are formed entirely by the bulk chains. (b) Probe chain (tethered chain) pulled at one end (tethered end) with velocity $V_{s}$ through immobile bulk chains. (c) Schematic showing the interfacial region of thickness $R_{0,1}$ and the slip length $\mathrm{b}=\mathrm{V}_{\mathrm{s}} / \kappa_{\mathrm{B}}$.

The picture in Figure $1 \mathrm{a}$ is equival ent to the case of a probe chain that is pulled by one end at a velocity $V_{s}$ through a stationary melt t,6 (see Figure 1b). Whereas the bulk experiences a shear rate $\kappa_{\mathrm{B}}$, the interfacial region experiences an effective shear rate $\kappa_{l}$ at the same wall shear stress. Depending on the magnitude of $\kappa_{1}, \mathrm{a}$ slip length b can be defined as shown in Figure 1c. We make an attempt in this paper to devel op a tube model that can quantitatively predict the interfacial shear rate $\kappa_{1}$, the slip velocity $V_{s}$ and the slip length $b$ at a given value of the bulk shear rate $\kappa_{\mathrm{B}}$ (or wall shear stress) with the knowledge of only molecular parameters.

The theoretical section is structured as follows. We begin by briefly summarizing the important equations of the CV model for the bulk chains. For a detailed description of the equations the reader is referred to the original CV model. ${ }^{7}$ We will then devel op a CV model for tethered chains. To do this we will first highlight the key differences between the dynamics of the bulk chains and the tethered chains. This physics is then incorporated into the equations of the CV model to derive the constitutive equation for tethered chains.

II.1. CV Model for Bulk. At steady-state conditions of simple shear flow, the constitutive equation for shear stress $\sigma_{12}$ consists of a set of coupled integro-differential equations given by ${ }^{7}$

$$
\begin{aligned}
& \sigma_{12, \mathrm{~B}}=\frac{15}{4} \mathrm{G}_{\mathrm{N}, \mathrm{B}} \frac{1}{\mathrm{~L}_{0, \mathrm{~B}}} \int_{-\mathrm{L}_{0, \mathrm{~B}} / 2}^{\mathrm{L}_{0, \mathrm{~B}} / 2} \mathrm{q}_{\mathrm{B}}{ }^{2} \mathrm{~S}_{12, \mathrm{~B}}\left(\mathrm{~s}_{0}\right) \mathrm{ds} \mathrm{s}_{0} \\
& \frac{\partial \mathrm{q}_{\mathrm{B}}\left(\mathrm{s}_{0}\right)}{\partial \mathrm{s}_{0}}=\frac{1}{6 \mathrm{PD}_{\text {rep }, \mathrm{P}}}\left[2 \frac{\left.\left\langle v_{\mathrm{B}}\right\rangle\right|_{\mathrm{s}_{0}=\mathrm{L}_{\mathrm{B}} / 2}}{\mathrm{~L}_{\mathrm{B}}}\right]\left(\mathrm{s}-\mathrm{s}_{0}\right)-\frac{\left\langle v_{\mathrm{B}}(\mathrm{s})\right\rangle}{3 \mathrm{P} \mathrm{D}_{\text {rep }, \mathrm{P}}}
\end{aligned}
$$




$$
\begin{aligned}
& \mathbf{S}_{\mathrm{B}}(\mathrm{s})=\int_{0}^{\infty} \frac{\partial \mathrm{G}_{\mathrm{B}}(\mathrm{s}, \theta)}{\partial \theta} \mathbf{Q}_{\mathrm{B}}\left[\mathbf{E}_{\mathrm{B}}(\theta)\right] \mathrm{d} \theta \\
& \frac{\partial \mathrm{G}_{\mathrm{B}}(\mathrm{s}, \theta)}{\partial \theta}=\mathrm{D}_{\text {rep }, \mathrm{P}} \frac{\partial^{2} \mathrm{G}_{\mathrm{B}}(\mathrm{s}, \theta)}{\partial \mathrm{s}^{2}}-\left\langle v_{\mathrm{B}}(\mathrm{s})\right\rangle \frac{\partial \mathrm{G}_{\mathrm{B}}(\mathrm{s}, \theta)}{\partial \mathrm{s}}-\mathrm{f}\left(\mathrm{q}_{\mathrm{B}}\right) \\
& {\left[2 \frac{\left.\left\langle v_{\mathrm{B}}\right\rangle\right|_{\mathrm{S}_{0}=\mathrm{L}_{\mathrm{B}} / 2}}{\mathrm{~L}_{\mathrm{B}}}\right] \mathrm{G}_{\mathrm{B}}(\mathrm{s}, \theta)-\frac{\mathrm{G}_{\mathrm{B}}(\mathrm{s}, \theta)}{\tau_{\xi, \mathrm{B}}(\mathrm{s})}}
\end{aligned}
$$

The various notations used in the above equations are as follows. Subscript B stands for bulk chains. $D_{\text {rep, }}=$ $L_{B}{ }^{2} /\left(\pi^{2} \tau_{d}(P)\right)$ is the longitudinal diffusivity of a bulk chain that has a reptation time of $\tau_{\mathrm{d}}(\mathrm{P}) . \mathrm{G}_{\mathrm{N}, \mathrm{B}}{ }^{0}$ is the high-frequency plateau modul us of the bulk chains. $L_{0, B}$ $=\mathrm{aP}$ is the equilibrium contour length of a bulk chain, where a is the tube diameter or the length of a segment. $\mathrm{S}_{12, \mathrm{~B}}$ is the shear component of the orientational order parameter tensor $\mathbf{S}_{\mathrm{B}}$. $\mathrm{S}_{0}$ is the undeformed reference coordinate of the contour length $\left(\mathrm{s}_{0}= \pm \mathrm{L}_{0, \mathrm{~B}} / 2\right.$ at the chain ends and $s_{0}=0$ at the center), while $s$ is the reference coordinate of the deformed contour length. $q_{B}$ is the segmental stretch ratio defined as $\mathrm{q}_{\mathrm{B}}=\partial \mathrm{s} / \partial \mathrm{s}_{0} \cdot\left\langle v_{\mathrm{B}^{-}}\right.$ $(\mathrm{s})\rangle=\kappa_{\mathrm{B}}: \int_{0}^{\mathrm{S}} \mathbf{S}_{\mathrm{B}}\left(\mathrm{s}^{\prime}\right) \mathrm{ds}$ is the continuous retraction velocity of a bulk chain al ong its contour and directed toward its center. ${ }^{13} \mathbf{E}_{\mathrm{B}}(\theta)$ is the deformation tensor for bulk chains, which for shear flow is simply $\mathrm{E}_{12, \mathrm{~B}}(\theta)=\kappa_{\mathrm{B}} \theta$ where $\theta$ is time. $\mathbf{Q}_{\mathrm{B}}$ is the geometric universal tensor defined as $\mathbf{Q}_{\mathrm{B}}=\left\langle\left(\mathbf{E}_{\mathrm{B}} \cdot \mathbf{u}\right)\left(\mathbf{E}_{\mathrm{B}} \cdot \mathbf{u}\right) /\left|\mathbf{E}_{\mathrm{B}} \cdot \mathbf{u}\right|\right\rangle\left\langle\left\langle\mathbf{E}_{\mathrm{B}} \cdot \mathbf{u} \mid\right\rangle-\mathbf{I} / 3\right.$. Here, \langle\rangle is the average over the isotropic distribution of unit vector $\mathbf{u}$ and $\mathbf{I}$ is the unit tensor. $\tau_{\xi, \mathrm{B}}$ is the fluctuation time scale of the bulk chains and is given by $\tau_{\xi, \mathrm{B}}(\mathrm{S})=\left(\tau_{\mathrm{r}}(\mathrm{P}) / 4\right) \exp \left(0.5 \vartheta \mathrm{N} \xi_{\mathrm{B}}{ }^{2}\right)$ where $\xi_{\mathrm{B}}=1-2|\mathrm{~s}| /$ $\mathrm{L}_{\mathrm{B}}, \vartheta=1.5$ and $\tau_{\mathrm{r}}(\mathrm{P})$ is the retraction time of the bulk chain. Finally, $\mathrm{G}_{\mathrm{B}}(\mathrm{s}, \theta)$ is the segment renewal probability for the bulk chain at time $\theta$ and at position $\mathrm{S}$.

The initial and boundary conditions for the segment renewal probability are

$$
\left.\begin{array}{ll}
\mathrm{G}_{\mathrm{B}}\left(\mathrm{s}_{0}\right)=1 \text { at } \theta=0 & \text { (initial condition) } \\
\mathrm{G}_{\mathrm{B}}(\theta)=0 \text { at } \mathrm{s}_{0}= \pm \mathrm{L}_{0, \mathrm{~B}} / 2 & \text { (due to fluctuations at chain end) } \\
\frac{\mathrm{dG}_{\mathrm{B}}(\theta)}{\mathrm{ds}_{0}}=0 \text { at } \mathrm{s}_{0}=0 & \text { (symmetry at the centre of chain) }
\end{array}\right\}
$$

II.2. CV Model for Tethered Chains. We will now develop the CV model for the case of tethered chains on the wall, which experience a shear rate $\kappa_{l}$. To begin with, it must be realized that there are several important differences between the modes of stress relaxation available to a flowing bulk chain and a stationary tethered chain. First at low shear rates, the dominant stress relaxation mechanism for a bulk chain is reptation, whereas a tethered chain cannot reptate at all. Second, constraint release (CR) becomes an important mechanism for relaxation of a tethered chain since the bulk chains, which form the tube of the tethered chain, can release the constraints on the tethered chain by reptation. This results in a local random jump in the conformation of the tethered chain, ${ }^{14}$ which relaxes its stress. On the other hand, the contribution of constraint release to stress relaxation of monodisperse bulk chains is negligible. ${ }^{12}$ Thus, constraint release (CR) becomes the principle mechanism of stress relaxation at low shear rates for a tethered molecule. The shear rates over which CR is active is given by $\kappa_{\mathrm{I}}<\tau_{\mathrm{CR}, \mathrm{I}}{ }^{-1}$, where $\tau_{\mathrm{CR}, \mathrm{I}}$ is the tube renewal time of the tethered chains due to constraint release by reptation of bulk chains. Short tethered chains containing $\mathrm{N}<\mathrm{N}_{\mathrm{c}}$, where $\mathrm{N}_{\mathrm{c}} \sim 10$, relax primarily by arm retraction at low shear rates. ${ }^{16}$ Thus, the relaxation mechanisms of a tethered chain are similar in many ways to those of an arm of a star polymer. ${ }^{4}$

In the shear rate range $\tau_{\mathrm{r}, I^{-1}}>\kappa_{\mathrm{l}}>\tau_{\mathrm{CR}, I^{-1}}$ the dominant relaxation mechanism is CCR, where $\tau_{r, l}$ is the retraction time of the tethered chain. In the CCR mechanism, the constraints on a test chain are rel eased when the end of a chain forming the tube is convected through the tube of the test chain. The convection velocity is determined by the motion along the contour that sets in by the continuous retraction process. For the case of a bulk chain the CCR relaxation occurs over the time scale of the order $\mathrm{O}\left(1 / \kappa_{\mathrm{B}}\right),{ }^{12}$ which is the same time scale over which the bulk chain is being deformed by flow. Hence the bulk chains can randomize their flowinduced orientation by CCR. However, if the test chain is a tethered molecule in the mushroom regime, the rate at which its constraints are released by CCR is determined by the convective velocity of the ends of the bulk chains, which form the tube of the tethered chain. Since the bulk chains experience a shear rate $\kappa_{B}$, the CCR relaxation time scale for a tethered chain is of the order $\mathrm{O}\left(1 / \kappa_{\mathrm{B}}\right)$. On the other hand, the time scale over which the tethered chain experiences deformation is of the order $\mathrm{O}\left(1 / \kappa_{1}\right)$. Thus, when $\kappa_{1} \gg \kappa_{\mathrm{B}}$, the tethered chains will preferentially orient in the flow direction. In other words, the "restricted" CCR in the vicinity of the wall is unable to prevent the orientation of the tethered chains. We postulate that this is the physical mechanism at a molecular level that is responsible for sudden orientation and slip.

Above the shear rate $\kappa_{l} \sim \tau_{r, 1}{ }^{-1}$, the chain relaxes mainly by fluctuations and partly by retraction. The contribution to stress comes from chain stretching. Tethering at one end slows down the retraction process. The retraction time for a tethered chain is four times larger than that of a free chain of the same molecular weight. ${ }^{14}$ The frequency of fluctuations of the contour length is maximum at the free end and decreases inward in such a way that there are no fluctuations at the tethered end.

Thus, a deformed tethered chain can relax its stress by four mechanisms: constraint release (CR) or arm retraction (AR) at low shear rates, restricted convective constraint release (CCR) at intermediate shear rates, and fluctuations and partial retraction at high shear rates. We will now incorporate this physical understanding in the mathematical framework of the CV model in order to devel op a constitutive equation for the interfacial region, which consists of tethered molecules grafted on the wall in the mushroom regime.

Let the undeformed reference coordinate of the contour length of the tethered chain be $\mathrm{s}_{0}$ with the first segment (tethered) at $\mathrm{s}_{0}=0$ and the free end at $\mathrm{s}_{0}=$ $L_{0,1}$. Here, $L_{0,1}$ is the equilibrium contour length of the tethered molecule. Let $\mathrm{s}$ be the contour length variable for a deformed tethered chain such that it varies from $\mathrm{s}=0$ at the tethered end to $\mathrm{s}=\mathrm{L}_{\mathrm{l}}$ at the free end. Then the segment renewal probability $\mathrm{G}_{\mathrm{I}}(\mathrm{s}, \theta)$ for the tethered chain at time $\theta$ and at position s under steady-state flow is given by 


$$
\begin{aligned}
& \frac{\partial \mathrm{G}_{1}(\mathrm{~s}, \theta)}{\partial \theta}=\mathrm{D}_{1} \frac{\partial^{2} \mathrm{G}_{1}(\mathrm{~s}, \theta)}{\partial \mathrm{s}^{2}}-\left\langle v_{1}(\mathrm{~s})\right\rangle \frac{\partial \mathrm{G}_{1}(\mathrm{~s}, \theta)}{\partial \mathrm{s}}- \\
& \mathrm{f}\left(\mathrm{q}_{1}\right)\left[2 \frac{\left\langle v_{2}\right\rangle}{\mathrm{L}_{\mathrm{B}}}\right] \mathrm{G}_{1}(\mathrm{~s}, \theta)-\frac{\mathrm{G}_{1}(\mathrm{~s}, \theta)}{\tau_{\xi, 1}(\mathrm{~s})}
\end{aligned}
$$

The first term on the right-hand side of eq 6 indicates tube renewal by constraint release. The diffusivity for the tethered chains can be written as ${ }^{15} D_{1}=2 L_{1}{ }^{2} /\left(\pi^{2} \tau_{1}\right)$. The dominating relaxation mode for $\mathrm{N}>\mathrm{N}_{\mathrm{C}}$ is $\mathrm{CR}$ so that $\tau_{1}=\tau_{\mathrm{CR}, \mathrm{I}}$ and the diffusion is controlled by CR process. For $\mathrm{N}<\mathrm{N}_{\mathrm{c}}$ the diffusion is due to arm retraction so that $\tau_{1}=\tau_{\mathrm{AR}, \mathrm{I}}{ }^{16} \mathrm{~F}$ or a tethered chain of $\mathrm{N}$ segments entangl ed with bulk chains of $\mathrm{P}$ segments, the $\mathrm{CR}$ time scale depends on the values of $\mathrm{N}$ and $\mathrm{P}$. The CR time scale for $N>N_{C}$ is given by ${ }^{4,16} \tau_{C R, I}(N) \approx$ $\mathrm{N}^{2}(\min (\mathrm{P}, \mathrm{N}))^{-0.5} \tau_{\mathrm{d}}(\mathrm{P})$. For $\mathrm{N}<\mathrm{N}_{\mathrm{c}}$, the arm retraction time is given by $\tau_{\mathrm{AR}, \mathrm{I}}(\mathrm{N}) \approx \mathrm{N}^{-1} \tau_{\mathrm{d}}(\mathrm{N}) \exp (\mathrm{VN})$, where $\tau_{\mathrm{d}}$ is the reptation time of a free chain with $\mathrm{N}$ segments and the numerical factor of $v$ is taken to be unity. The value of critical number of segments $\mathrm{N}_{\mathrm{c}}$ does not exceed 10.16

The second term in eq 6 indicates convection of the mesh relative to the chain at an average relative velocity $\left\langle v_{1}(\mathrm{~s})\right\rangle$ between the chain and its mesh. As explained later in eq $12,\left\langle v_{1}(\mathrm{~s})\right\rangle$ consists of two components namely, the retraction velocity of the tethered chain along its contour and the slip velocity $\mathrm{V}_{\mathrm{s}}$.

The third term in eq 6 gives the tube renewal probability due to CCR wherein the constraints on the tethered chain are released by convection of the chain end of bulk chains at an average velocity $\left\langle v_{2}\right\rangle$ that arises due to continuous retraction. $\left\langle v_{2}\right\rangle$ is defined later in eq 13. The prefactor $f\left(q_{1}\right)$ in the third term is the switch function, which is used to reduce the dominance of the CCR when the corresponding segment is stretched. We have used here the self-consistent switch function described by Mead et al., ${ }^{7}$ viz, $f\left(q_{1}\right)=q_{1}{ }^{-1}$. Here, $q_{1}$ is the local stretch of the tethered chain $\left(q_{1}=d s / d s_{0}\right)$.

The last term in eq 6 describes the fluctuation contribution to tube renewal, which is governed by the fluctuation time scale $\tau_{\xi, \mathrm{I}}\left(\mathrm{S}_{0}\right)$ given by

$$
\tau_{\xi, l}(\mathrm{~s})=\left(4 \tau_{\mathrm{r}}(\mathrm{N})\right) \exp \left(\vartheta \mathrm{N} \xi_{1}^{2}\right)
$$

where $\xi_{1}=1-\left|\mathrm{s}\left(\mathrm{S}_{0}\right)\right| / \mathrm{L}_{1}$ and $\vartheta$ is 1.5. The prefactor of 4 multiplying the retraction time $\tau_{\mathrm{r}}$ of $\mathrm{N}$ chain accounts for the effect of tethering one end of the chain. ${ }^{14}$

At steady state, the segmental stretch $q_{1}$ is given by

$$
\frac{\partial \mathrm{q}_{1}\left(\mathrm{~s}_{0}\right)}{\partial \mathrm{s}_{0}}=\frac{1}{6 \mathrm{ND} D_{\text {rep }, \mathrm{N}}}\left[2 \frac{\left\langle v_{2}\right\rangle}{\mathrm{L}_{\mathrm{B}}}\right]\left(\mathrm{s}-\mathrm{s}_{0}\right)-\frac{\left\langle v_{1}\left(\mathrm{~s}_{0}\right)\right\rangle}{3 \mathrm{ND}_{\mathrm{rep}, \mathrm{N}}}
$$

where $q_{1}=1 \ldots$ at $s_{0}=L_{0,1}$.

In eq 8, $D_{\text {rep, } N}=L_{1}{ }^{2} /\left(\pi^{2} \tau_{d}(N)\right)$ is the reptative diffusion coefficient and $\tau_{\mathrm{d}}(\mathrm{N})\left(=3 \mathrm{~N} \tau_{\mathrm{r}}(\mathrm{N})\right)$ is the reptation time of a free chain with $\mathrm{N}$ segments.

The initial and boundary conditions for the tube renewal probability in eq 6 can be arrived at from the following discussion. It can be seen from eq 7 that the magnitude of fluctuations decreases exponentially from the free end to the tethered end. Since the maximum fluctuations at the free end do not allow the endsegment to remain in the tube, $\mathrm{G}_{\mathbb{1}}(\theta)=0$ at the free end. Also, since the other tube renewal mechanisms namely, CR and CCR, are uniform over the entire tube length, while the fluctuations are negl igible at the tethered end, the boundary condition at the tethered end should be $\mathrm{dG}_{\mathrm{I}}(\theta) / \mathrm{ds}_{0}=0$. Thus, the initial and boundary conditions are

$$
\left\{\begin{array}{l}
\mathrm{G}_{\mid}\left(\mathrm{s}_{0}\right)=1 \text { at } \theta=0 \\
\mathrm{G}_{\mid}(\theta)=0 \text { at } \mathrm{s}_{0}=\mathrm{L}_{0, \mathrm{~B}} \\
\frac{\mathrm{dG}_{\mid}(\theta)}{\mathrm{ds}_{0}}=0 \text { at } \mathrm{s}_{0}=0
\end{array}\right\}
$$

The net relative vel ocity between the segments of the tethered chain and its tube consists of two components: the first component is a result of the continuous retraction motion of the deforming tethered chain as given by ${ }^{13}$

$$
\left\langle v_{1}\left(\mathrm{~s}_{0}\right)\right\rangle=\kappa_{1}: \int_{0}^{\mathrm{s}} \mathbf{S}_{1}\left(\mathrm{~s}^{\prime}\right) \mathrm{d} \mathrm{s}^{\prime}
$$

$\mathbf{S}_{\mid}\left(\mathrm{s}^{\prime}\right)$ in eq 10 is the orientational order parameter tensor for the tethered chain given by

$$
\mathbf{S}_{\mid}\left(\mathrm{s}_{0}\right)=\int_{0}^{\infty} \frac{\partial \mathbf{G}_{1}\left(\mathrm{~s}_{0}, \theta\right)}{\partial \theta} \mathbf{Q}_{\mid}\left[\mathbf{E}_{1}(\theta)\right] \mathrm{d} \theta
$$

Here $\mathbf{E}_{l}(\theta)$ is the deformation tensor for the tethered chains, which for shear flow is given by $\mathbf{E}_{12, \mathrm{I}}=\kappa_{\mathrm{l}} \theta$. $\mathbf{Q}_{\text {। }}$ is the geometric universal tensor defined as $\mathbf{Q}_{\mathbf{l}}=\left\langle\left(\mathbf{E}_{\mathbf{l}}\right.\right.$. $\left.\mathbf{u})\left(\mathbf{E}_{1} \cdot \mathbf{u}\right) /\left|\mathbf{E}_{1} \cdot \mathbf{u}\right|\right\rangle\left\langle\left\langle\mathbf{E}_{1} \cdot \mathbf{u} \mid\right\rangle-\mathbf{I} / 3\right.$. \langle\rangle is the average over the isotropic distribution of unit vector $\mathbf{u}$, and $\mathbf{I}$ is the unit tensor.

The second component of the relative velocity between the tethered chain segments and their corresponding tube segments arises from the macroscopic velocity $V_{s}$ of the tethered chain relative to the stationary tube formed by bulk chains (see Figure 1b). Since the retraction velocity $\left\langle v_{1}\right\rangle$ of eq 10 is always positive, ${ }^{17}$ the direction of relative movement of the segments is al ways toward the tethered end, which is parallel to the direction of the slip vel ocity $V_{\mathrm{s}}$. Hence the net average relative velocity between the tethered molecule and its mesh is given by

$$
\left\langle v_{1}\left(\mathrm{~s}_{0}\right)\right\rangle=\mathrm{V}_{\mathrm{s}}+\left\langle v_{1}\left(\mathrm{~s}_{0}\right)\right\rangle
$$

As discussed earlier, the relaxation of tethered chains due to CCR is determined by the velocity of the end segments of the bulk chains. This velocity is cal culated as

$$
\left\langle v_{2}\right\rangle=\left\langle v_{\mathrm{B}}\left(\mathrm{s}_{0}\right)\right\rangle=\kappa_{\mathrm{B}}: \int_{0}^{\mathrm{L}_{\mathrm{B}} / 2} \mathbf{S}_{\mathrm{B}}\left(\mathrm{s}^{\prime}\right) \mathrm{ds^{ \prime }}
$$

where the bulk shear rate $\kappa_{\mathrm{B}}$ and the integral are to be calculated from the constitutive equation for bulk chains (eqs $1-5$ ) at the same shear stress as that experienced by the tethered chains.

The shear stress in the interfacial region is given by

$$
\sigma_{12, \mathrm{I}}=\frac{15}{4} \mathrm{G}_{\mathrm{N}, \mathrm{I}} \frac{1}{\mathrm{~L}_{0, \mathrm{I}}} \int_{0}^{\mathrm{L}_{0, \mathrm{l}}} \mathrm{q}_{\mathrm{I}}{ }^{2} \mathrm{~S}_{12, \mathrm{I}}\left(\mathrm{s}_{0}\right) \mathrm{ds}_{0}
$$

where $G_{N, 1}{ }^{0}$ is the plateau modulus of the interfacial region at the wall. The calculation of the stress in the interfacial region from eq 14 requires an additional relationship between the slip vel ocity $V_{s}$ and the effective shear rate experienced by the tethered molecules $\kappa_{1}$. We use a simple relationship in analogy to that proposed earlier by Wang, ${ }^{18}$ 


$$
\mathrm{V}_{\mathrm{s}}=\mathrm{R}_{0,1} \kappa_{\mathrm{l}}
$$

where $R_{0,1}=a \sqrt{N}$ is equilibrium end-to-end distance of a tethered molecule, which is also equal to the thickness of the grafted layer on the wall in the mushroom regime. ${ }^{19}$ This assumption can be expected to remain valid as long as the tethered chain does not stretch extensively. Thus, we can expect overprediction of $\mathrm{V}_{\mathrm{s}}$ after strong slip.

Besides the slip vel ocity, one also requires knowledge of the plateau modulus of the interfacial region, which is expected to depend on the surface coverage of the tethered chains. By definition, the plateau modulus is proportional to the entanglement density, which for tethered chains in mushroom regime, can be calculated as follows. Consider a small volume of height approximately equal to the entanglement spacing a shown by the dotted lines in Figure la. Let there be $v$ chains grafted on this area such that the chains per unit area are $\Sigma=$ v/area. Then the number of entanglements (or segments) in this volume element of height $a$ is $\mathrm{v} /(\mathrm{a} \times$ area $)=\Sigma / \mathrm{a}$. Consequently, the plateau modulus is given by

$$
\mathrm{G}_{\mathrm{N}, \mathrm{l}}{ }^{0}=\frac{\Sigma}{\mathrm{N}_{\mathrm{A}} \mathrm{a}} \mathrm{RT}
$$

where $N_{A}$ is the Avogrado number, $R$ is the universal gas constant, and $\mathrm{T}$ is the absolute temperature. Substituting eq 16 into eq 14 gives

$$
\sigma_{12, \mathrm{I}}=\frac{15}{4} \frac{\sum \mathrm{kT}}{\mathrm{a}}\left\{\frac{1}{\mathrm{~L}_{\mathrm{ol}}} \int_{0}^{\mathrm{L}_{\mathrm{ol}}} \mathrm{q}_{\mathrm{l}}{ }^{2} \mathrm{~S}_{12, \mathrm{I}}\left(\mathrm{s}_{0}\right) \mathrm{ds} \mathrm{s}_{0}\right\}
$$

Equation 17 is the constitutive equation for deforming tethered chains. Finally, there is one more equation that couples the dynamics of the interfacial and bulk regions. This equation is the continuity of shear stress at the interface as given by

$$
\sigma_{12, \mathrm{~B}}=\sigma_{12, \mathrm{I}}
$$

In the above discussi on, we have presented a contour variable (CV) tube model for tethered chains. The model consists of the coupled integro-differential eqs 1-18 that must be solved simultaneously for predicting the flow curves in the interfacial region at the wall. The CV model for tethered chains accounts for the following important physics: In the low shear rate regime, constraint rel ease or arm retraction replaces reptation as the dominant relaxation mechanism. An additional relative velocity arising from the slip velocity exists between the segments of the tethered chain and their tube. The retraction velocity of the bulk chain ends restricts the convective constraint release process for tethered chain. Finally, the tethered chains have a slower retraction time relative to a free chain of same molecular weight. The model suggests that the restricted convective constraint release experienced by the tethered chains would naturally give rise to a nonmonotonic flow curve.

The model parameters are: the number of entanglements per chain for the bulk molecules $(P)$, the number of entanglements per chain for the tethered molecules $(\mathrm{N})$, the reptation time $\left(\tau_{\mathrm{d}}\right)$ of the bulk chain, the plateau modulus of the bulk $\mathrm{G}_{\mathrm{N}, \mathrm{B}}{ }^{0}$ and the surface coverage $(\Sigma)$. The first two parameters require the knowledge of entanglement molecular weight $\mathrm{M}_{\mathrm{e}}$, which has been well documented in the literature. ${ }^{23}$ The next two parameters can be determined from independent rheological experiments and are also documented in the literature for several polymers. ${ }^{20}$ The last parameter $(\Sigma)$ can be controlled by modifying the polymer-surface chemistry. The other time scales that appear in the set of equations, namely, reptation, CR, and retraction, can be estimated from the various scaling relationships described elsewhere.4,14,16 The wall shear stress in all of the calculations presented bel ow has been nondimensionalized using the bulk plateau modulus. Similarly, the slip vel ocity has been nondimensi onalized using the reptation time of the bulk chain and the equilibrium contour length of the bulk chain unless specified otherwise. Thus, $\mathrm{P}, \mathrm{N}$, and $\Sigma$ are the only parameters required to obtain the solution.

The detailed procedure for solving the integro-differential constitutive equations of the type described here can be found el sewhere. $7,8,17,21$ H owever, the solution of the above developed model is more complicated because of the fact that the constitutive equations for the bulk and the interfacial regions are coupled. The coupling arises from the fact that the retraction velocity required for the CCR relaxation of tethered chains has to be calculated from the constitutive equation of bulk while simultaneously maintaining the stress continuity between the two regions. The numerical procedure required to solve the coupled equations is briefly described below. F or a given value of $\kappa_{l}$, the value of shear stress in the interfacial region is initially predicted from eqs 6 to 14 assuming $\left\langle v_{2}\right\rangle=0 .\left\langle v_{2}\right\rangle$ is then calculated from the bulk constitutive equation at the same shear stress by inverting eqs $1-5$ numerically. Next, this value of $\left\langle v_{2}\right\rangle$ is now used to solve the constitutive equation for the interfacial region to predict a new value of the stress. This procedure is repeated until the shear stress and $\left\langle v_{2}\right\rangle$ converges between previous and current iterations. An alternative procedure would be to first choose a value of the bulk shear rate $\kappa_{\mathrm{B}}$, then calculate $\left\langle v_{2}\right\rangle$ and stress in the bulk using eqs $1-5$. Next, the interfacial shear rate $\kappa_{l}$ and, consequently, the slip vel ocity are calculated by using eqs 6-14 for a range of values of $\kappa_{\mathrm{B}}$ so that the interfacial stress matches with the bulk stress. In either procedure a numerical inversion of the integro-differential equations is required. We find that the above numerical procedure is robust until a high value of interfacial shear rate $\left(\kappa_{1}>1 / \tau_{r, 1}\right)$ after which the convergence is seriously affected due to the change of the nature of equations from parabolic to hyperbolic. It is important to note that the input variables to the problem are either the bulk shear rate or the wall shear stress, while the predicted variables are the interfacial shear rate, the slip velocity and the slip length. Thus, the input variables are the same as the control variables used in extrusion (or simple shear) experiments and the predicted variables are the same as those measured from experiments.

\section{Predictions}

All calculations presented in this section have been carried out for the case of a poly(dimethylsiloxane) (PDMS) melt, for which very precise experimental data on wall slip is available from the recent work of Leger and co-workers. ${ }^{19,22-24}$ The plateau modulus of PDMS melt is known to be $0.18 \mathrm{MPa}, 20$ its molecular weight between entanglements $M_{e}$ is $13522 \mathrm{~kg} / \mathrm{kg} \mathrm{mol}, 20$ and the segment length between entanglements can be estimated to be $a=7.855 \mathrm{~nm}$. 


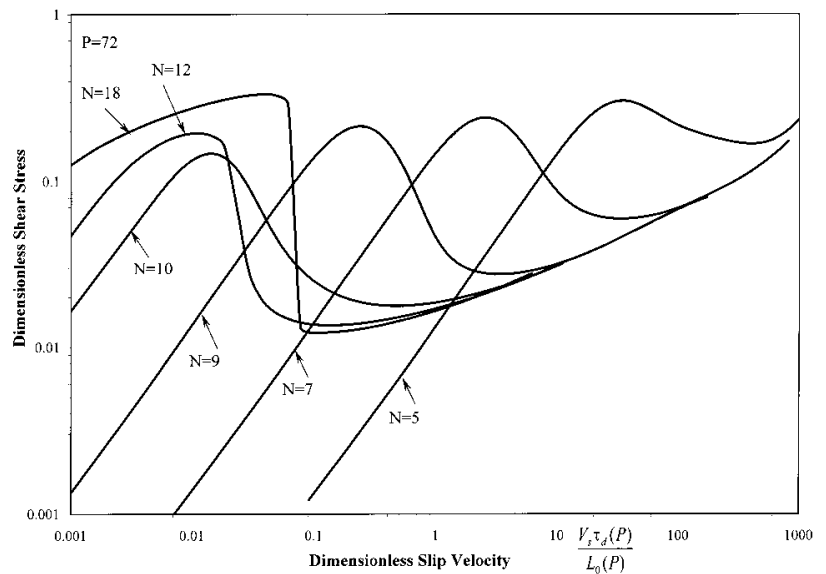

Figure 2. Dimensionless shear stress plotted against dimensionless slip velocity for various values of $N$, keeping $P$ constant. It can be seen that as $\mathrm{N}$ decreases the severity of nonmonotonicity and the jump in slip velocity decreases.

III.1. Flow Curves. We will first examine the flow curves of the tethered chains and the influence of molecular weight of the tethered chains on the flow curves. In Figure 2 the molecular weight of the bulk chain is kept constant while the mol ecular weight of the tethered chain is varied. The nondimensional shear stress is plotted against nondimensional slip velocity for various values of $N$ while keeping constant $P=72$ and surface coverage $\Sigma=3 \times 10^{17} \mathrm{~m}^{-2}$. This choice of surface coverage essentially renders the interfacial plateau modulus equal to that of the bulk and has been chosen arbitrarily to demonstrate the main predictions of the model. However, all of the trends in Figure 2 are observed for other values of $\Sigma$ also. The flow curve for the bulk chains shows a slight nonmonotonic nature for $1 / \tau_{\mathrm{d}, \mathrm{B}}<\kappa_{\mathrm{B}}<1 / \tau_{\mathrm{r}, \mathrm{B}}$ for the high value of $\mathrm{P}=72$. The small amount of nonmonotonicity is in agreement with the nature of the CV model and indicates that a further relaxation mechanism is still missing in this shear rate range. An improvement of the CCR mechanism has been recently proposed, ${ }^{25}$ which is expected to make the bulk flow curve fully monotonic.

For the tethered chains, however, it can be seen that the shear stress becomes increasingly nonmonotonic as $\mathrm{N}$ increases. This nonmonotonic nature implies the occurrence of an instability in which the slip velocity can discontinuously increase and thereby cause a stickslip transition. Clearly, such instability has its origin in the interfacial region close to the wall. The maximum in the flow curve corresponds to a critical wall shear stress and a critical slip velocity (or a corresponding critical shear rate) at which the instability sets in. Figure 2 further shows that the shear stress increases monotonically in the low shear rateregion $\kappa_{1} \tau_{\mathrm{cr}, \mathrm{l}}=\mathrm{V}_{\mathrm{s}} \tau_{\mathrm{cr}, \mathrm{l}} /$ $\mathrm{R}_{0,1}<1$, indicating that a deformed tethered chain relaxes faster (due to CR or AR) than the rate at which it is deformed. Also, in the high shear rate regi on above $\mathrm{V}_{\mathrm{s}} \tau_{r, 1} / \mathrm{R}_{0,1} \sim 1$ the contour length of the chain starts increasing, and consequently, the stress increases monotonically with shear rate due to stretching. In the intermediate shear rate region, however, the shear stress becomes nonmonotonic for high $\mathrm{P}$ and $\mathrm{N}$ values. In this region the main relaxation mechanism is CCR. Clearly, the CCR is ineffective in completely randomizing the orientation of the tethered chains. This happens, as proposed earlier, because the CCR time scale, which is determined by the retraction velocity of bulk
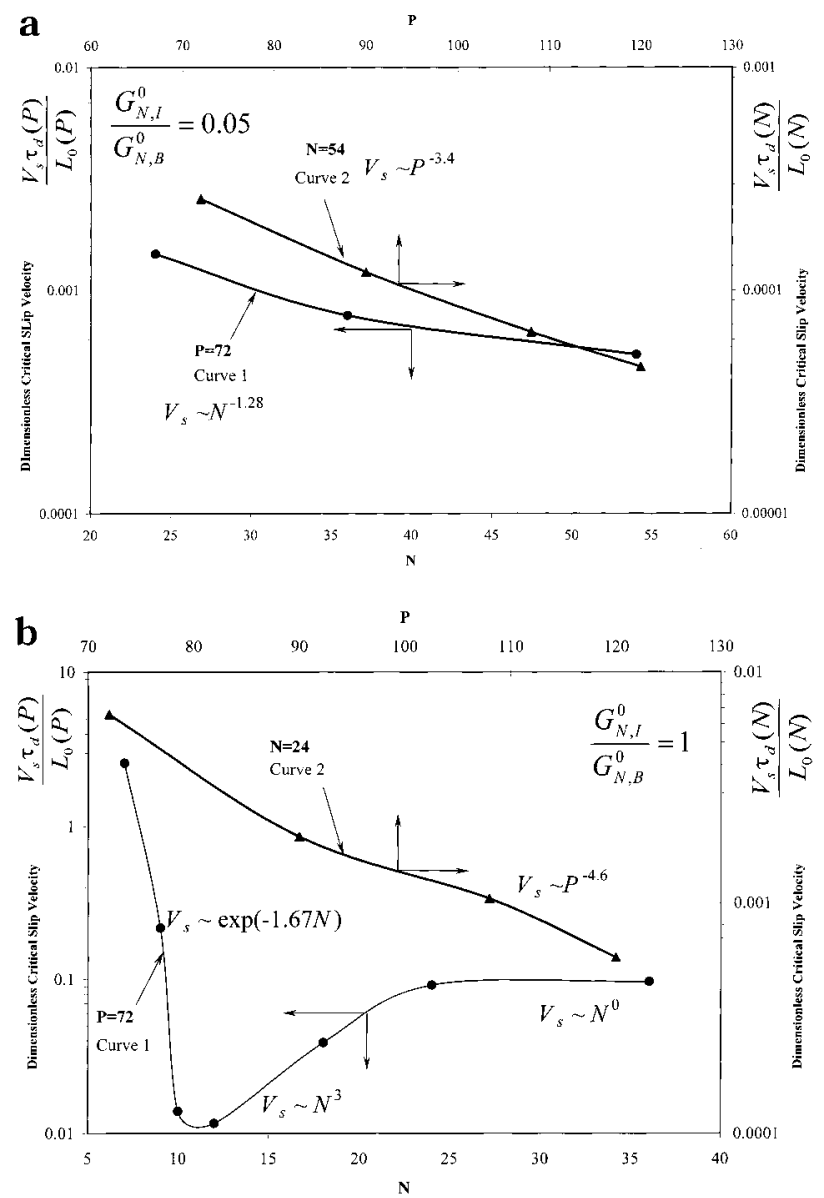

Figure 3. Critical slip velocity (at the onset of instability) is plotted against number segments of tethered and bulk chain for two values of plateau modulus ratio: (a) $\mathrm{G}_{\mathrm{N}, 1} \mathrm{O} / \mathrm{G}_{\mathrm{N}, \mathrm{B}}{ }^{0}=0.05$, and (b) $\mathrm{G}_{\mathrm{N}, \mathrm{I}^{0}} / \mathrm{G}_{\mathrm{N}, \mathrm{B}}{ }^{0}=1$.

chains is longer than the time scale over which the tethered chains experience deformation $\left(\kappa_{1} \tau_{\mathrm{CCR}, \mathrm{I}} \sim \kappa_{\mathrm{l}} \mathrm{L}_{\mathrm{B}} /\right.$ $\left\langle v_{2}\right\rangle \sim \kappa_{l} / \kappa_{\mathrm{B}}>1$ ). Thus, the restricted CCR relaxation of the tethered chains cannot prevent their flow-induced orientation above the critical shear rate. As a result, the bulk chains suddenly lose their entanglements with the tethered chains and slip with a high slip velocity.

The critical shear rate at which the flow curve becomes nonmonotonic decreases with increase in $\mathrm{N}$ due to the slowing down of the CR or the AR relaxation with molecular weight. For very small values of $\mathrm{N}$ the fluctuations of the contour length relax the orientation of the chain. The relaxation due to fluctuations is highly effective in low molecular weight chains compared to that in high molecular weight chains. ${ }^{26}$ This means that the low molecular weight tethered chains are less susceptible to orientation than the higher molecular weight tethered chains. As a result, the nonmonotonic nature of the flow curve decreases with decreasing $\mathrm{N}$.

III.2. Scaling. If $\tau$ is a typical relaxation time of the tethered chain, then the orientation of the chain would begin when the Weissenberg number $\dot{\gamma}_{1} \tau \sim 1$. Thus, if the dominant relaxation mode were constraint release then

$$
\mathrm{V}_{\mathrm{s}}^{*}=\left\langle\mathrm{R}_{\mathrm{o}}^{2}\right\rangle^{1 / 2} \dot{\gamma}_{\mathrm{I}} \sim \frac{\left\langle\mathrm{R}_{\mathrm{o}}{ }^{2}\right\rangle^{1 / 2}}{\tau_{\mathrm{CR}, \mathrm{I}}} \sim \frac{\mathrm{N}^{0.5}}{\mathrm{P}^{3} \mathrm{~N}^{1.5}}=\mathrm{P}^{-3} \mathrm{~N}^{-1}
$$

This scaling law is demonstrated in Figure $3 a$ for the 


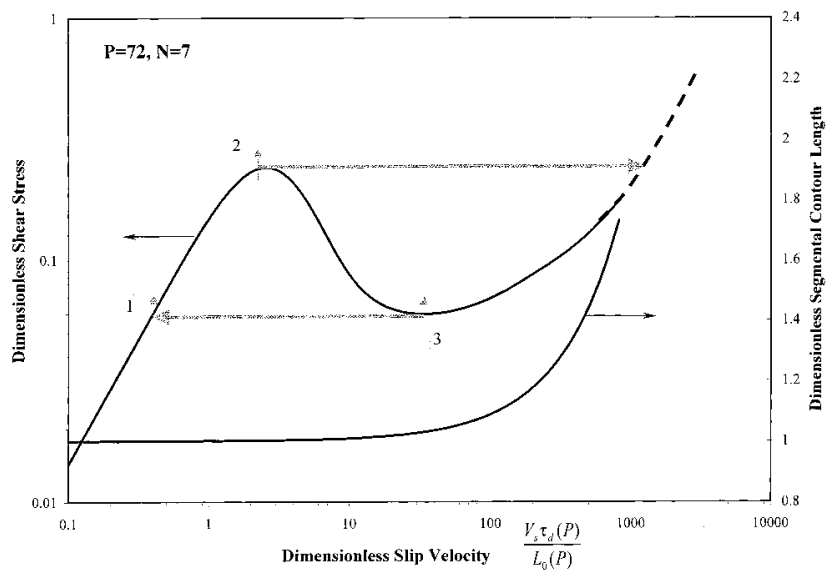

Figure 4. Dimensi onless shear stress and segmental contour length are plotted against dimensionless slip velocity for $\mathrm{P}=$ 72 and $\mathrm{N}=7$. A hysteresis loop in the stress-controlled mode can be seen. The part of the curve with a negative slope indicates instability

case of $\mathrm{G}_{\mathrm{N}, \mathrm{I}} \% \mathrm{G}_{\mathrm{N}, \mathrm{B}} \mathrm{B}^{0}=0.05$ and $\mathrm{N}<\mathrm{P}$. It agrees with that predicted by the Brochard et al. scaling model. ${ }^{5}$ Leger et al.22 have experimentally validated this scaling, although in their experiments $\mathrm{N} \geq \mathrm{P}$ for which the above scaling should in fact be replaced by

$$
\mathrm{V}_{\mathrm{s}}{ }^{*}=\left\langle\mathrm{R}_{\mathrm{o}}{ }^{2}\right\rangle^{1 / 2} \dot{\gamma}_{1} \sim \frac{\left\langle\mathrm{R}_{\mathrm{o}}{ }^{2}\right\rangle^{1 / 2}}{\tau_{\mathrm{CR}, \mathrm{I}}} \sim \frac{\mathrm{N}^{0.5}}{\mathrm{P}^{2.5} \mathrm{~N}^{2}} \sim \mathrm{P}^{-2.5} \mathrm{~N}^{-1.5}
$$

For the case of $\mathrm{N}<\mathrm{N}_{c} \sim 10$, arm retraction is dominating mode of relaxation ${ }^{16}$ and the scaling for the critical slip velocity can be given by

$$
\mathrm{V}_{\mathrm{s}}{ }^{*}=\left\langle\mathrm{R}_{\mathrm{o}}{ }^{2}\right\rangle^{1 / 2} \dot{\gamma}_{1} \sim \frac{\left\langle\mathrm{R}_{\mathrm{o}}^{2}\right\rangle^{1 / 2}}{\tau_{\mathrm{I}}} \sim \frac{\mathrm{N}^{0.5}}{\mathrm{~N}^{2} \mathrm{e}^{\mathrm{N}}} \sim \mathrm{N}^{-1.5} \mathrm{e}^{-\mathrm{N}}
$$

F or higher values of $G_{N, 1} / G_{N, B}{ }^{0}$ (i.e., for higher surface coverage), the dependence of the critical slip vel ocity on $\mathrm{P}$ and $\mathrm{N}$ becomes complicated as shown in Figure 3b. This is possibly due to the combined effect of the different relaxation mechanisms, which interact to produce a scaling, that does not agree with any one of the specific relaxation mechanisms. Scaling laws for very short and very long tethered chains have not been explored experimentally so far and hence direct comparisons are not possible.

The critical stress for stick-slip is predicted by eq 17 to be directly proportional to the surface coverage and temperature. This is in agreement with the Brochardde Gennes model ${ }^{3}$ and has also been observed experimentally. ${ }^{18}$

III.3. Coil-to-Stretch Transition. In Figure 4, we plot the shear stress and contour length of the tethered molecule against slip velocity for the case of $P=72$ and $\mathrm{N}=7$. For simplicity, we divide the plot in three regions, first in which the stress increases with slip vel ocity, the second, in which the stress decreases with slip velocity, and the third, in which the stress again increases. In the third region, the numerical scheme adopted in this work poses some difficulties with respect to convergence at high slip velocities due to the change in the nature of the partial differential equation (eq 6) from parabolic to hyperbolic. The dashed line in this region simply indicates the possible trend in the increase in shear stress at the high slip velocities. In a controlled stress

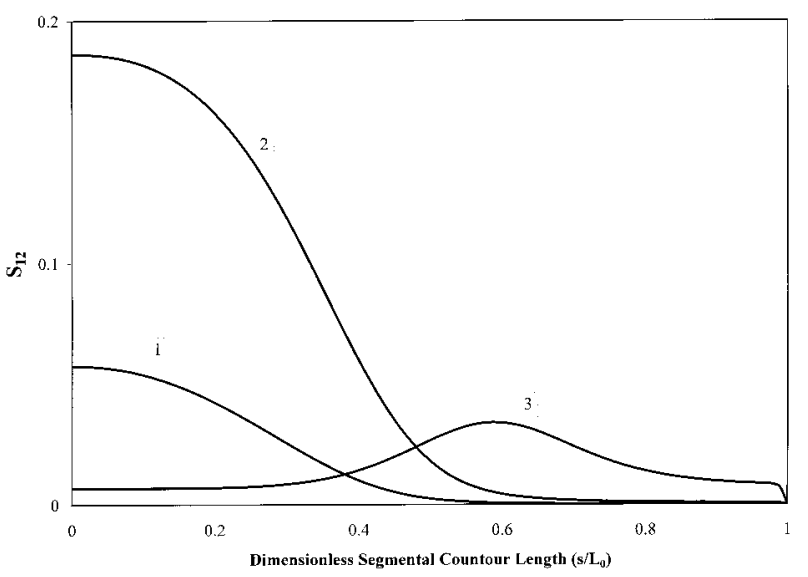

Figure 5. Shear component of the orientational order parameter tensor is plotted against the segmental contour length variable. Curves 1-3 correspond to various locations described on the shear stress-slip velocity curve of Figure 4.

extrusion experiment, the thick arrows in Figure 4 indicate the hysteresis associated with the slip instability. For a rate-controlled extrusion process, the second region of negative slope will correspond to an unstable region, where flow and pressure oscillations are expected to prevail. It is interesting to see that in this region while the stress becomes nonmonotonic, the contour length of the tethered molecule increases by a factor of only about two. The end-to-end distance of the tethered chain should also obviously increase with the orientation of the molecule. Thus, the jump in slip velocity is associated with a "coil-to-stretch" transition in which the tethered chain suddenly increases its endto-end distance at a critical shear stress without increasing its segmental contour length substantially.

In Figure 5, the shear component of the orientational order parameter tensor $\mathrm{S}_{12}$ is plotted against the nondimensionalized length scale al ong the segmental contour length $\mathrm{S}_{0} / \mathrm{L}_{0}$ for various locations $1-3$ on the shear stress curve of Figure 4. For the corresponding curves in Figure $5, \mathrm{~S}_{12}$ is al ways zero at $\mathrm{s}_{0} / \mathrm{L}_{0}=1$. This implies that the segmental orientation is al ways random at the chain end, which is due to the fluctuations of the last segments. For all curves 1-3 in Figure 5, the value of $\mathrm{S}_{12}$ increases toward the tethered end indicating that the orientation increases near the tethered end. Point 1 in Figure 4 is in the region where AR is the dominating relaxation mechanism. The molecule has little orientation at this point and hence shows low values of $\mathrm{S}_{12}$ in the corresponding curve in Figure 5. At point 2 in Figure 4, the value of $S_{12}$ attains a maximum at the tethered end, which consequently leads to maximum stress. Further increase in shear rate after the stress maximum, corresponding to point 3 in Figure 4, shows a decrease in the value of $\mathrm{S}_{12}$ as seen in the curve 3 of Figure 5. This implies a strong orientation of the segments near the tethered end. Any further increase in shear rate increases the contour length since $\kappa_{l}>$ $\tau_{r, l^{-1}}$. This picture of the orientation of a tethered molecule is very similar to that proposed by Ajdari et al. ${ }^{4}$ and Mhetar and Archer. ${ }^{6}$

III.4. Comparison with Experimental Data. We now compare the predictions of our model with the experimental data of Léger and co-workers. ${ }^{19,22-24}$ These experiments were performed using monodisperse endgrafted PDMS chains of molecular weight $96000 \mathrm{~kg} / \mathrm{kg}$ mol that are grafted on a wall to give a fractional surface 


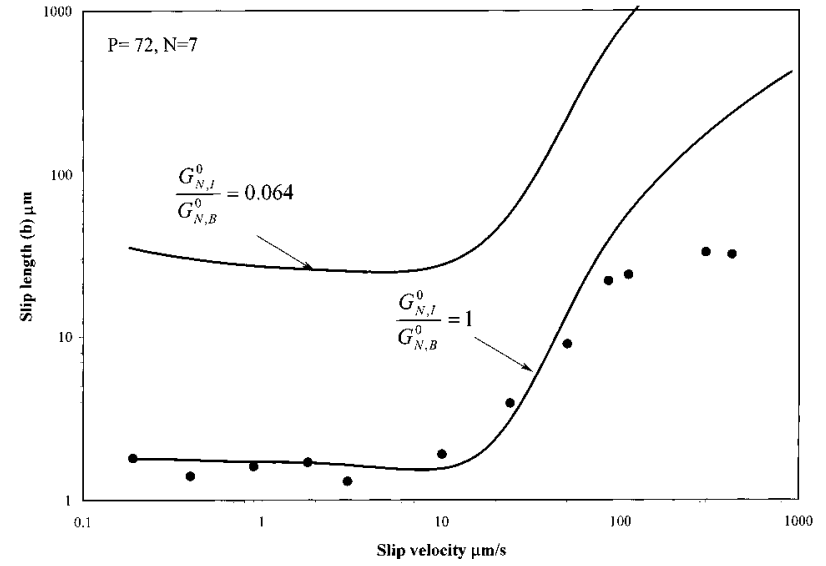

Figure 6. Comparison of model prediction with experimental data of Léger and co-workers ${ }^{23}$ for a PDMS melt of $\mathrm{P} \sim 72$ flowing past a wall on which PDMS chains of $\mathrm{N} \sim 7$ are endtethered. The upper curve corresponds to $\mathrm{G}_{\mathrm{N}, 1^{0}}=0.0115 \mathrm{MPa}$ (i.e., $\mathrm{G}_{N, 1}{ }^{0} / \mathrm{G}_{N, B}{ }^{0}=0.064$ ) while the lower corresponds to $\mathrm{G}_{N, I^{0}}$ $=\mathrm{G}_{\mathrm{N}, \mathrm{B}}{ }^{0}=0.18 \mathrm{MPa}$ for which a good fit with the experimental data can be seen.

coverage of 0.0055 , i.e, an actual surface coverage of $\Sigma$ $=0.0055 /\left(5 \times 10^{-10}\right)^{2}=2.2 \times 10^{16}$ chains $/ \mathrm{m}^{2}$. The grafted wall is in contact with a monodisperse melt of molecular weight $970000 \mathrm{~kg} / \mathrm{kg}$ mol. Velocities were measured at a distance of $70 \mathrm{~nm}$ from the wall, which is of the order of end-to-end distance of the bulk chains $\left(\left\langle R_{0, B}\right\rangle^{1 / 2}=67 \mathrm{~nm}\right)$. The values of the various model parameters for PDMS have been listed earlier. At the experimental surface coverage of $\Sigma=2.2 \times 10^{16}$ chains/ $\mathrm{m}^{2}$, the interfacial plateau modulus takes the value of $0.0115 \mathrm{MPa}$ according to eq 16.

Figure 6 shows the slip length $\left(b=V_{s} / \kappa_{B}\right)$ plotted against slip velocity for two values of the interfacial plateau modulus namely, $\mathrm{G}_{\mathrm{N}, 1}{ }^{0}=0.0115 \mathrm{MPa}$ and $\mathrm{G}_{\mathrm{N}, \mathrm{I}^{0}}$ $=\mathrm{G}_{\mathrm{N}, \mathrm{B}}{ }^{0}=0.18 \mathrm{MPa}$. The bulk shear rate for calculating $b$ is taken as that value where the shear stress in the bulk is equal to the shear stress in the interfacial region (eq 18). The points in Figure 6 show the experimental data while the lines shows the tube model prediction for the two values of the interfacial plateau modulus. The model prediction of the critical slip velocity has been corrected to account for the fact that the experimental fractional surface coverage of 0.0055 is actually greater than the overlap fractional surface coverage of 0.00077 by nearly a factor of 7 . F or surface coverage above the overlap value, the bulk chains still penetrate the grafted layer sufficiently to avoid entanglements between the tethered chains. ${ }^{19,23}$ Our model is thus, in principle, applicable at this surface coverage with a minor modification to the constraint release time scale. This modification is done by realizing that in this regime of surface coverage the critical slip velocity increases linearly with the surface coverage, while the slip length essentially remains locked at the value corresponding to that at the overlap coverage. 5,19,23 Thus, the critical slip velocity predicted in the mushroom regime (below overlap) should increase by the same factor of 7, while the slip length should be the same as that predicted at overlap. This amounts to merely shifting the slip length predicted in the mushroom regime horizontally by the factor of 7. The lines in Figure 6 show the predictions of the model when these corrections are applied. It can be seen that the model almost quantitatively predicts the experimental critical slip velocity data.
The present model, however, substantially overpre dicts the critical slip length if the interfacial plateau modulus value calculated from eq 16 were to be used. The predicted slip length matches with the experimental data only for a higher value of the interfacial plateau modulus. This is because as the number of entanglements in the interfacial region increase, the shear stress that can be borne by the tethered chains at a given slip velocity increases, thereby increasing the bulk shear rate and consequently decreasing the slip length. Thus, it appears that the actual number of entanglements in the interfacial region is far more than that calculated from eq 16. Another possible reason for the overprediction of slip length could be that the bulk rheological behavior of the slightly polydisperse PDMS of $\mathrm{P}=72$ might not be accurately predicted by $\mathrm{CV}$ model. Only the zero-shear viscosity data for this polymer was available, whereas for a fair comparison, the complete rheogram would be needed.

Figure 6 also shows that above the critical slip velocity, the model overpredicts the slip length. The reason for this is most probably the fact that eq 15 is not expected to be valid after strong slip because of the strong orientation of the tethered molecules. I nterestingly, the slip length before the critical slip velocity is of the order of 2 to $3 \mu \mathrm{m}$, which when compared to the coil size of the tethered molecule $\left\langle\mathrm{R}_{0,1^{2}}\right\rangle^{1 / 2}=0.0209 \mu \mathrm{m}$, clearly indicates that the bulk chains have a finite nonzero slip velocity even before the strong slip. Thus, there is a "weak-slip" region before the strong-slip region such that the no-slip boundary condition fails even before the strong-slip sets in. This behavior is in qualitative agreement with that observed recently by Munstedt et al. ${ }^{27}$ for the extrusion of polyethylene through a slit die.

\section{Conclusions and Discussions}

We have developed a molecular model for tethered chains in the mushroom region. Our model considers all possible modes of relaxation applicable to a tethered chain namely, constraint release, convective constraint release, fluctuations, and retraction. The model predicts a discontinuous strong slip that arises from sudden disentanglement of the tethered chains from the flowing bulk chains. The predictions of the scaling laws for critical slip velocity and the molecular features of the coil-stretch transition from our model are in agreement with previous scaling models. 3,4,6 However, the most important prediction of our model that differentiates it from other previous models is that tethered polymer chains experience a suppressed CCR relaxation; because of this, they can be oriented by shear flow above a critical shear rate and critical shear stress. This, we believe, is the main molecular mechanism that drives strong slip by sudden disentanglement and has not been recognized in any of the previous scaling models for slip. Our model also differs from previous scaling models by virtue of its potential ability to quantitatively predict slip parameters based solely on molecular information.

There are several other important predictions of the model. The tendency to show stick-slip instability increases with molecular weight of the bulk molecules and of the tethered molecules. Shorter tethered molecules do not orient easily compared to longer molecules. Thus, extrusion of a material containing small molecular weight chains or extrusion through a die coated with small chains will not show a stick-slip instability. The 
scaling laws predicted by the model for the critical stress and the critical slip velocity agree with those predicted by the earlier scaling models. However, our model also predicts different scaling laws for cases of very low and very high $\mathrm{N}$. The model predicts that the stick-slip transition is caused by a coil-to-stretch transition of the tethered molecules, which is also in agreement with the earlier scaling models. ${ }^{3}$ Our model quantitatively predicts the critical slip velocity at which strong slip occurs, but requires much larger number of entangl ements per unit volume in the interfacial region in order to quantitatively match the experimental slip length in the weak-slip region. This issue needs further exploration. The model also predicts two regions for the slip length, a weak-slip region for low shear rates and a strong-slip region for high shear rates. Thus, the model suggests that the conventional no-slip boundary condition may not be valid for a large range of extrusion shear rates.

Finally, it is important to mention the limits within which our present model is applicable. We have so far considered the specific case of monodispersed bulk and end-tethered chains. In a real system, both the bulk and tethered chains would have a distribution in chain lengths. The relaxation due to CR and CCR would be faster in a polydisperse material due to presence of shorter chains. This would decrease the time scale for the randomization process of the tethered molecules and thereby prevent their orientation. Thus, polydispersity would decrease the nonmonotonicity of flow curve as observed experimentally. ${ }^{28} \mathrm{~A}$ real system is further complicated by the fact that a single bulk molecule can tether to multiple sites on the solid wall, giving rise to loops and tails. We have not addressed the dynamics of the loops and their influence on slip in this paper. The present tube model needs to be further refined to account for polydispersity and dynamics of loops on slip. The present model also does not consider the effects of other mol ecular architectural parameters such as longchain branching. We hope, however, that the new molecular insights that we have provided in the wall slip phenomenon will stimulate further theoretical and experimental work in this fascinating field.

Acknowledgment. Y.M.J . would like to acknowledge the financial assistance from Council of Scientific and Industrial Research, SRF grant. The authors are also grateful to Prof. G. Marrucci and Dr. G. Ianniruberto for an insightful remark on our approach, which helped us improve the analysis substantially.

\section{References and Notes}

(1) Bergam, N. Proceedings of the VIIth International Congress on Rheology; Stockholm, Sweden, 1976; p 50.

(2) J oshi, Y. M.; Lele, A. K.; Mashelkar, R. A. J . Non-Newtonian Fluid Mech. 2000, 89, 303-335.

(3) Brochard, F.; de Gennes, P. G. Langmuir 1992, 8, 3033-3037.

(4) Ajdari, A. F.; Brochard-Wyart, F.; de Gennes, P. G.; Leibler, L.; Viovy, J . L.; Rubenstein, M. Physica A 1994, 204, 17-39.

(5) Brochard-Wyart, F.; Gay C.; de Gennes, P. G. Macromol ecules 1996, 29, 377-382.

(6) Mhetar, V. R.; Archer, L. A. Macromol ecules 1998, 31, 66396649.

(7) Mead, D. W.; Larson, R. G.; Doi, M. Macromolecules 1998, 31, 7895-7914.

(8) Marrucci, G.; Grizzuti, N. Gazz. Chim. Ital. 1988, 118, 179185.

(9) Lin, Y.-H. J . Rheol. 1985, 29, 605-637.

(10) Mcleish, T. C. B.; Ball, R. C. J . Polym. Sci.: Part B 1986, 24, 1735-1745.

(11) Yang, X.; Wang, S.-Q.; Halasa, A.; Ishida, H. Rheol. Acta 1998, 37, 415-423.

(12) Marrucci, G. J . Non-Newtonian Fluid Mech. 1996, 62, 279289.

(13) Doi, M.; Edwards, S. F. The theory of polymer dynamics; Oxford University Press: London, 1986.

(14) Graessley, W. W. Adv. Polym. Sci. 1982, 47, 67-117.

(15) Watanabe, H. Prog. Polym. Sci. 1999, 24, 1253-1403.

(16) Brochard-Wyart, F.; Ajdari, A.; Leibler, L.; Rubinstein, M.; Viovy, J. L. Macromolecules 1994, 27, 803-808.

(17) Mead, D. W.; Yavich, D.; Leal, L. G. Rheol. Acta 1995, 34, 339-359.

(18) Wang, S.-Q. Adv. Polym. Sci. 1999, 138, 227-275.

(19) Léger, L.; Raphael, E.; Hervet, H. Adv. Polym. Sci. 1999, 138, 185-226.

(20) Fetters, L. J .; Lohse, D. J .; Richter, D.; Witten, T. A.; Zirke, A. Macromolecules 1994, 27, 4639-4647.

(21) Mead, D. W.; Leal, L. G. Rheol. Acta. 1995, 34, 360-383.

(22) Léger, L.; Hervet, H.; Massey, G. In Rheology for Polymer Melt Processing; Piau, J .-M., Agassant, J .-F., Eds.; Elsevier Science: Amsterdam, 1996; p 337.

(23) Durliat, E.; Hervet, H.; Léger, L. Europhys. Lett. 1997, 38, 383-388.

(24) Léger, L.; Hervet, H.; Auroy, P.; Boucher, E.; Massey, G. In Rheol ogy for Polymer Melt Processing; Piau, J .-M., Agassant, J .-F., Eds.; Elsevier Science: Amsterdam, 1996; $p 1$.

(25) Ianniruberto, G.; Marrucci, G. XIIIth I nternational Congress on Rheol ogy; Binding, D. M., Hudson, N. E., Mewis, J ., Piau, J .-M., Petrie, C. J . S., Townsend, P., Wagner, M. H., Walters, K., Eds.; 2000; Vol 2, pp 102-104.

(26) I anniruberto, G.; Marrucci, G. J . Non-Newtonian Fluid Mech. 2000, 95, 363-374.

(27) Munstedt, H.; Schmidt, M.; Wassner, E. J . Rheol. 2000, 44, 413-427.

(28) Myerholtz, R. W. J . Appl. Polym. Sci. 1967, 11, 687-698.

MA0010200 